\title{
Constraints on BSM physics through the Higgs couplings
}

\author{
Jérémie Quevillon* \\ King's College London, Physics Department, Strand, London WC2R 2LS, U.K \\ E-mail: jeremie.quevillon@kcl.ac.uk
}

\begin{abstract}
We discuss the implications from the measured mass and production/decay rates of the observed Higgs boson on several well motivated extensions of the Standard Model.

Firstly, we demonstrate that the value $M_{h} \simeq 125 \mathrm{GeV}$ together with the non-observation of superparticles at the LHC, indicates that the SUSY-breaking scale $M_{S}$ is rather high, $M_{S}>1 \mathrm{TeV}$, leading to a Higgs sector that can be described, to a good approximation, by only two free parameters. we then show that in the Minimal Supersymmetric Standard Model, to a good approximation, the phenomenology of the lighter Higgs state can be described by its mass and three couplings: those to massive gauge bosons and to top and bottom quarks. We also address the promising Heavy scalar searches at the LHC.
\end{abstract}

Secondly, we discuss how the LHC limits on the invisible Higgs branching fraction impose strong constraints on Higgs portal models of dark matter.

Frontiers of Fundamental Physics 14 - FFP14,

15-18 July 2014

Aix Marseille University (AMU) Saint-Charles Campus, Marseille

${ }^{*}$ Speaker. 


\section{The post-Higgs boson discovery MSSM Higgs sector}

In the MSSM, two Higgs doublets $H_{d}$ and $H_{u}$ are needed to break the electroweak symmetry, leading to three neutral and two charged Higgs states. The tree-level masses of the CP-even $h$ and $H$ bosons depend only on $\tan \beta=v_{d} / v_{u}$, the ratio of vevs of the two doublets and on the pseudoscalar Higgs mass $M_{A}$. Nevertheless, many parameters of the MSSM such as the SUSY scale, taken to be the geometric average of the stop masses $M_{S}=\sqrt{m_{\tilde{t}_{1}} m_{\tilde{t}_{2}}}$, the higgsino mass $\mu$ and the stop/bottom trilinear couplings $A_{t / b}$ enter $M_{h / H}$ through loop corrections. The CP-even Higgs mass matrix can be written in the basis as:

$$
\mathscr{M}_{S}^{2}=M_{Z}^{2}\left(\begin{array}{cc}
c_{\beta}^{2} & -s_{\beta} c_{\beta} \\
-s_{\beta} c_{\beta} & s_{\beta}^{2}
\end{array}\right)+M_{A}^{2}\left(\begin{array}{cc}
s_{\beta}^{2} & -s_{\beta} c_{\beta} \\
-s_{\beta} c_{\beta} & c_{\beta}^{2}
\end{array}\right)+\left(\begin{array}{cc}
\Delta \mathscr{M}_{11}^{2} & \Delta \mathscr{M}_{12}^{2} \\
\Delta \mathscr{M}_{12}^{2} & \Delta \mathscr{M}_{22}^{2}
\end{array}\right)
$$

where we use the notation $c_{\beta} \equiv \cos \beta, s_{\beta} \equiv \sin \beta$ and include the radiative corrections into a $2 \times 2$ matrix $\Delta \mathscr{M}_{i j}^{2}$. One can then easily derive the Higgs masses $M_{h, H}$ and the mixing angle $\alpha$ that diagonalizes the $h, H$ system, $h=-\sin \alpha H_{d}^{0}+\cos \alpha H_{u}^{0}$ and $H=\cos \alpha H_{d}^{0}+\sin \alpha H_{u}^{0}$ :

$$
\begin{aligned}
M_{h / H}^{2} & =\frac{1}{2}\left(M_{A}^{2}+M_{Z}^{2}+\Delta \mathscr{M}_{11}^{2}+\Delta \mathscr{M}_{22}^{2} \mp \sqrt{M_{A}^{4}+M_{Z}^{4}-2 M_{A}^{2} M_{Z}^{2} c_{4 \beta}+C}\right) \\
\tan \alpha & =\frac{2 \Delta \mathscr{M}_{12}^{2}-\left(M_{A}^{2}+M_{Z}^{2}\right) s_{\beta}}{\Delta \mathscr{M}_{11}^{2}-\Delta \mathscr{M}_{22}^{2}+\left(M_{Z}^{2}-M_{A}^{2}\right) c_{2 \beta}+\sqrt{M_{A}^{4}+M_{Z}^{4}-2 M_{A}^{2} M_{Z}^{2} c_{4 \beta}+C}} \\
C= & 4 \Delta \mathscr{M}_{12}^{4}+\left(\Delta \mathscr{M}_{11}^{2}-\Delta \mathscr{M}_{22}^{2}\right)^{2}-2\left(M_{A}^{2}-M_{Z}^{2}\right)\left(\Delta \mathscr{M}_{11}^{2}-\Delta \mathscr{M}_{22}^{2}\right) c_{2 \beta}-4\left(M_{A}^{2}+M_{Z}^{2}\right) \Delta \mathscr{M}_{12}^{2} s_{2 \beta}
\end{aligned}
$$

In previous works $[1,2,3,4]$, it was pointed out that since the measured value of the $h$ boson mass is high, $M_{h}=125 \mathrm{GeV}$, leading to a rather large SUSY-breaking scale [5], $M_{S} \gtrsim 1 \mathrm{TeV}$, it implies that the leading radiative corrections are now almost fixed when the constraint $M_{h}=$ $125 \mathrm{GeV}$ is taken into account. In the $2 \times 2$ correction matrix of eq. (1.1), only the $\Delta \mathscr{M}_{22}^{2}$ entry which involves the by far leading top/stop corrections proportional to the fourth power of the top Yukawa coupling, is relevant to a good approximation [6]. In this limit $\Delta \mathscr{M}_{22}^{2} \gg \Delta \mathscr{M}_{11}^{2}, \Delta \mathscr{M}_{12}^{2}$, one can simply trade $\Delta \mathscr{M}_{22}^{2}$ for the known $M_{h}$ value:

$$
\Delta \mathscr{M}_{22}^{2}=\frac{M_{h}^{2}\left(M_{A}^{2}+M_{Z}^{2}-M_{h}^{2}\right)-M_{A}^{2} M_{Z}^{2} c_{2 \beta}^{2}}{M_{Z}^{2} c_{\beta}^{2}+M_{A}^{2} s_{\beta}^{2}-M_{h}^{2}} .
$$

In this case, called hMSSM in Ref.[6], one obtains simple expressions for the mass $M_{H}$ and the angle $\alpha$ in terms of $M_{A}, \tan \beta$ and $M_{h}$ :

$$
\begin{aligned}
M_{H}^{2} & =\frac{\left(M_{A}^{2}+M_{Z}^{2}-M_{h}^{2}\right)\left(M_{Z}^{2} c_{\beta}^{2}+M_{A}^{2} s_{\beta}^{2}\right)-M_{A}^{2} M_{Z}^{2} c_{2 \beta}^{2}}{M_{Z}^{2} c_{\beta}^{2}+M_{A}^{2} s_{\beta}^{2}-M_{h}^{2}} \\
\operatorname{hMSSM}: \quad & =-\arctan \left(\frac{\left(M_{Z}^{2}+M_{A}^{2}\right) c_{\beta} s_{\beta}}{M_{Z}^{2} c_{\beta}^{2}+M_{A}^{2} s_{\beta}^{2}-M_{h}^{2}}\right) .
\end{aligned}
$$

Concerning the charged Higgs boson, the quantum corrections to its mass are much smaller for large $M_{A}$, and one can write to a good approximation, $M_{H^{ \pm}}^{2} \simeq M_{A}^{2}+M_{W}^{2}$.

This approach allows to disregard the radiative corrections in the MSSM Higgs sector and their complicated dependence on all the MSSM parameters. This considerably simplifies the phenomenological studies in the MSSM Higgs sector which up to now do not use the constraint $M_{h}=125 \mathrm{GeV}$ as an input as it should be, and rely either on benchmark scenarios in which most of the MSSM parameters are fixed or refuge to large scans over the parameter space. 


\section{Fit of the SM Higgs couplings}

In the MSSM, the couplings of the lighter $h$ state to gauge bosons and fermions, normalized to their SM values read:

$$
c_{V}^{0}=\sin (\beta-\alpha), \quad c_{t}^{0}=\frac{\cos \alpha}{\sin \beta}, c_{b}^{0}=-\frac{\sin \alpha}{\cos \beta} .
$$

They depend on the tree-level inputs $\tan \beta$ and $M_{A}$ but also on the full MSSM spectrum because of the quantum corrections that enter the angle $\alpha$ as in the case of the Higgs masses. As discussed earlier, knowing $\tan \beta$ and $M_{A}$ and fixing $M_{h}$ to its measured value, the couplings can be determined. Nevertheless, this applies only for the radiative corrections to the Higgs masses. In addition, there exists direct radiative corrections to the Higgs couplings different from the ones of the mass matrix in eq. (1.1) and which will complicate the situation.

If the $h$ coupling to the bottom and top quarks could be significantly modified (by stop loops in the production process $g g \rightarrow h$ in the former and by the $\Delta_{b}$ corrections in the latter cases; see Ref.[6]), $c_{t, b}^{0} \rightarrow c_{t, b}$, the couplings to $\tau$ leptons and $c$ quarks do not receive substantial direct corrections and one still has $c_{c, \tau} \approx c_{t, b}^{0}$. Consequently, because of the direct radiative corrections, the Higgs couplings cannot be described by only $\beta$ and $\alpha$ as in eq. (2.1). To characterize the Higgs particle at the LHC, it was advocated [6] that three independent $h$ couplings should be considered, namely $c_{t}, c_{b}$ and $c_{V}=c_{V}^{0}$. Thus, one can define the following effective Lagrangian:

$$
\mathscr{L}_{h}=c_{V} g_{h V V} h V_{\mu}^{+} V^{-\mu}+c_{t} y_{t} h \bar{t}_{L} t_{R}-c_{t} y_{c} h \bar{c}_{L} c_{R}-c_{b} y_{b} h \bar{b}_{L} b_{R}-c_{b} y_{\tau} h \bar{\tau}_{L} \tau_{R}+\text { h.c. }
$$

where $y_{t, c, b, \tau}=m_{t, c, b, \tau} / v$ are the Yukawa couplings of the heavy SM fermions, $g_{h V V}=2 M_{V}^{2} / v$ the $h V V$ couplings with $V=W, Z$. Following an earlier analysis performed in Ref.[7] where details can be found, a three-dimensional fit of the $\sqrt{s}=7+8 \mathrm{TeV}$ ATLAS and CMS Higgs data has been performed and the result in the space $\left[c_{t}, c_{b}, c_{V}\right]$ is shown on the left-hand side of Fig. 1. The obtained best-fit values for the Higgs couplings are: $c_{t}=0.89, c_{b}=1.0$ and $c_{V}=1.02$.

In cases where the direct corrections are not quantitatively significant one can reduce the number of effective parameters down to two using the MSSM relations of eq. (2.1). Using the formulae of eq. (1.5) for the mixing angle and the $M_{h} \approx 125 \mathrm{GeV}$ value as an input, one can perform a fit in the $\left[\tan \beta, M_{A}\right]$ plane as shown on the right-hand side of Fig. 1. It illustrates the $68 \%$, 95\% and $99 \% \mathrm{CL}$ contours obtained from fitting the signal strengths and their ratios. The best-fit point is realized for the values $\tan \beta=1$ and $M_{A}=557 \mathrm{GeV}$, which translates into $M_{H}=580 \mathrm{GeV}$, $M_{H^{ \pm}}=563 \mathrm{GeV}$ and $\alpha=-0.837 \mathrm{rad}$. Such a low $\tan \beta$ point implies an extremely large SUSY scale value, $M_{S}=\mathscr{O}(100) \mathrm{TeV}$ to accommodate a $125 \mathrm{GeV}$ Higgs boson. Notice, that the $\chi^{2}$ value is relatively flat all over the $1 \sigma$ region and, thus, larger $\tan \beta$ values could also be appropriate, hence allowing for not too large SUSY scale values. Nevertheless, one obtains that the pseudoscalar should verify $M_{A} \gtrsim 200 \mathrm{GeV}$ in all cases.

\section{Heavy scalar searches}

In our quite "model-independent" approach, defined in eq. (1.5), we make no restriction on the SUSY scale which can be at any value, even quite high. It allows to reopen the small $\tan \beta$ 

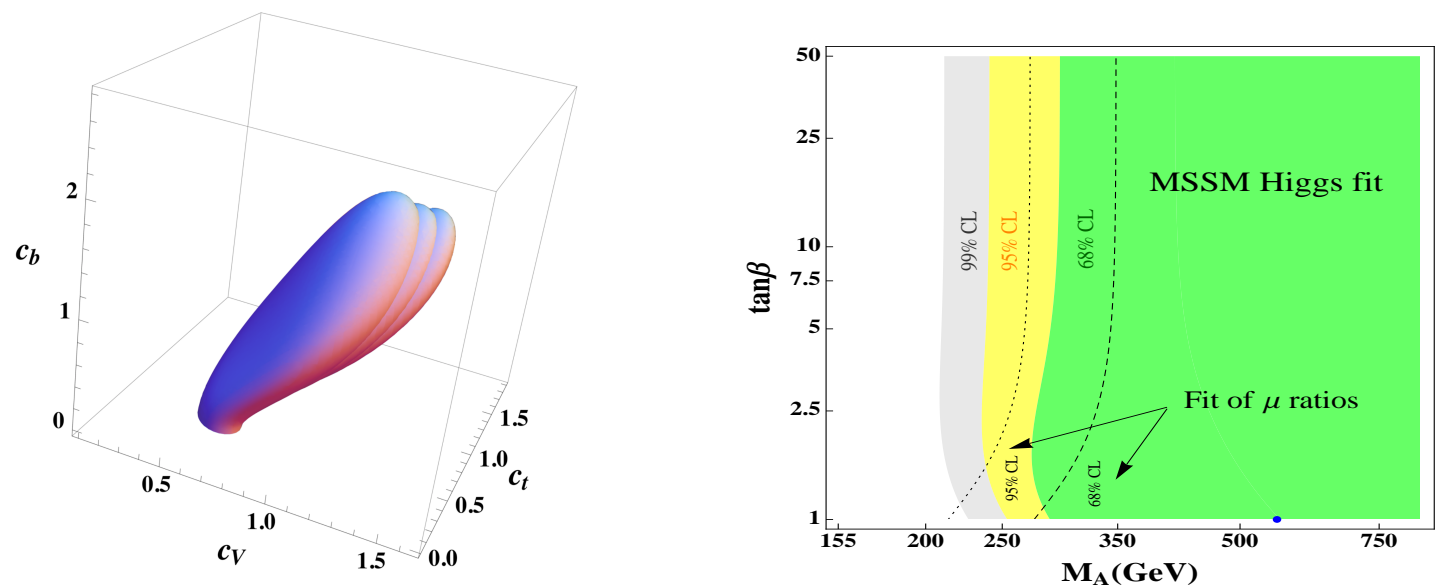

Figure 1: Left: best-fit regions at 99\%CL for the Higgs signal strengths in the three dimensional space $\left[c_{t}, c_{b}, c_{V}\right]$ [6]. Right: best-fit regions for the signal strengths and their ratios in the plane $\left[\tan \beta, M_{A}\right]$; the best point is in blue [6].

region, $\tan \beta \lesssim 3$, that was long thought to be excluded from the negative search of a SM-like scalar boson at LEP which set the limit $M_{h} \gtrsim 114 \mathrm{GeV}$, but assuming a setting with $M_{S} \lesssim 1 \mathrm{TeV}$. If $M_{S}$ is large enough as indicated by present data (see Ref.[5] for example), low $\tan \beta$ values would still be allowed. In the left-hand side of Fig. 2, we display the contours in the plane $\left[\tan \beta, M_{S}\right]$ for mass values in the window $M_{h}=120-132 \mathrm{GeV}$ of the observed Higgs state.

The contour corresponding to the LEP2 limit $M_{h}=114 \mathrm{GeV}$ indicates that $\tan \beta \approx 1$ is still viable provided that $M_{S} \gtrsim 20 \mathrm{TeV}$. The present value $M_{h}=125$ sets stronger constraints: for example, while one can accommodate a scale $M_{S} \approx 1 \mathrm{TeV}$ with $\tan \beta \approx 5$, a large scale $M_{S} \approx 20 \mathrm{TeV}$ is required to obtain $\tan \beta \approx 2$. Let us discuss the implications for heavy Higgs searches.

The most promising process to look for the heavier MSSM Higgs scalars is by far $p p \rightarrow$ $g g+b b \rightarrow H / A \rightarrow \tau \tau$. Searches for this channel have been performed by ATLAS [8] with $\approx 5$ $\mathrm{fb}^{-1}$ data at the $7 \mathrm{TeV}$ run and by CMS [9] with $\approx 5+20 \mathrm{fb}^{-1}$ data at the $7 \mathrm{TeV}$ and $8 \mathrm{TeV}$ runs. Upper limits on the production cross section times decay branching ratio have been set and they can be turned into constraints on the MSSM parameter space. The sensitivity of the CMS $p p \rightarrow h, H, A \rightarrow \tau \tau$ analysis in the plane $\left[\tan \beta, M_{A}\right]$ using $25 \mathrm{fb}^{-1}$ of data can be found in Ref.[9]. The excluded region obtained from the observed limit at the 95\% CL is extremely restrictive and for $M_{A} \approx 250 \mathrm{GeV}$ the high $\tan \beta \gtrsim 10$ region is entirely excluded and one is even sensitive to large values $M_{A} \approx 800 \mathrm{GeV}$ for $\tan \beta \gtrsim 45$.

Nevertheless, there is a caveat to this exclusion limit because the constraint applies for a particular benchmark, the maximal mixing scenario with $X_{t} / M_{S}=\sqrt{6}$, assuming $M_{S}=1 \mathrm{TeV}$. In fact this exclusion limit is valid in far more situations than the "MSSM $M_{h}^{\max }$ scenario" and it should be extended to the low $\tan \beta$ regime which, in the chosen scenario with $M_{S}=1 \mathrm{TeV}$, is excluded by the LEP2 limit on the lighter $h$ mass but is resurrected if the SUSY scale is kept as a free parameter. Reopening the low $\tan \beta$ region allows to hunt for the heavier scalar bosons in various interesting processes at the LHC. Heavier CP-even $H$ decays into massive gauge bosons $H \rightarrow W W, Z Z$ and lighter Higgs bosons $H \rightarrow h h, \mathrm{CP}-$ odd scalar decays into a vector and a Higgs boson, $A \rightarrow h Z$, 
$\mathrm{CP}-$ even and $\mathrm{CP}-$ odd scalar decays into top quarks, $H / A \rightarrow t \bar{t}$, and the charged scalar decays into a gauge boson and a Higgs boson, $H^{ \pm} \rightarrow W h$.

A preliminary study of these processes has been performed [2] relying on the searches for the SM Higgs boson or other heavy resonances made by the ATLAS and CMS collaborations. The results which are shown on the left-hand of Fig. 2 are interesting since these searches cover a large part of the parameter space of the MSSM Higgs sector in a model-independent way, i.e. without the need to precise the SUSY particle spectrum that appear in the quantum corrections. More especially, the channels $H \rightarrow V V$ and $H / A \rightarrow t \bar{t}$ are very constraining as they probe the entire low $\tan \beta$ area up to $M_{A} \approx 600 \mathrm{GeV}$. Notice that $A \rightarrow h Z$ and $H \rightarrow h h$ could also be seen at the current LHC in small parts of the MSSM parameter space.
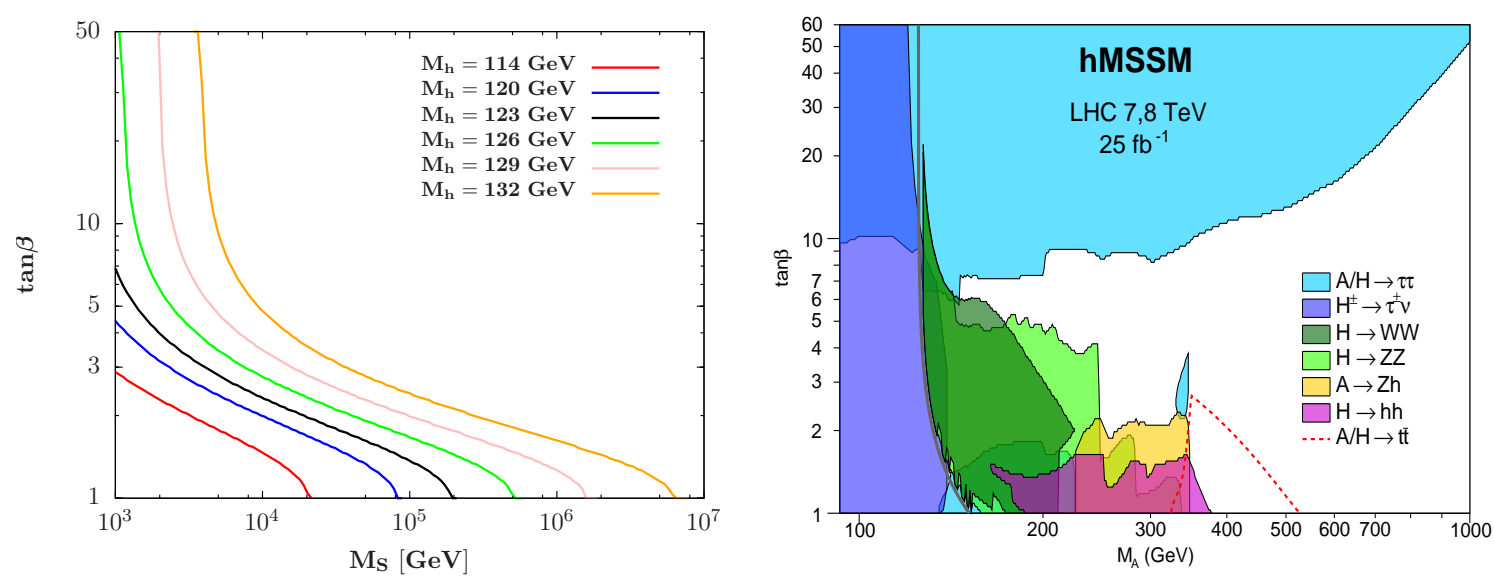

Figure 2: Left: contours for fixed values $M_{h}=120-132 \mathrm{GeV}$ in the $\left[\tan \beta, M_{S}\right]$ plane in the decoupling limit $M_{A} \gg M_{Z}$; the "LEP2 contour" for $M_{h}=114 \mathrm{GeV}$ is shown in red. Right: the estimated sensitivities in the various search channels for the heavier MSSM Higgs bosons in the $\left[\tan \beta, M_{A}\right]$ plane: $H / A \rightarrow \tau \tau$, $H \rightarrow W W+Z Z, H / A \rightarrow t \bar{t}, A \rightarrow h Z$ and $H \rightarrow h h$. Taken from Ref.[4].

\section{LHC searches for Higgs-portal dark matter}

If the invisible particle into which the Higgs boson decays is a constituent of dark matter in the universe, the Higgs coupling to dark matter can be probed not only at the LHC but also in direct detection experiments. In this section, we discuss the complementarity of these two direct detection methods. We consider generic Higgs-portal scenarios in which the dark matter particle is a real scalar, a real vector, or a Majorana fermion, $\chi=S, V, f[10]$. The relevant terms in the effective Lagrangian in each of these cases are

$$
\begin{aligned}
& \Delta \mathscr{L}_{S}=-\frac{1}{2} m_{S}^{2} S^{2}-\frac{1}{4} \lambda_{S} S^{4}-\frac{1}{4} \lambda_{h S S} H^{\dagger} H S^{2}, \\
& \Delta \mathscr{L}_{V}=\frac{1}{2} m_{V}^{2} V_{\mu} V^{\mu}+\frac{1}{4} \lambda_{V}\left(V_{\mu} V^{\mu}\right)^{2}+\frac{1}{4} \lambda_{h V V} H^{\dagger} H V_{\mu} V^{\mu}, \\
& \Delta \mathscr{L}_{f}=-\frac{1}{2} m_{f} f f-\frac{1}{4} \frac{\lambda_{h f f}}{\Lambda} H^{\dagger} H f f+\text { h.c. }
\end{aligned}
$$

The partial Higgs decay width into dark matter $\Gamma(H \rightarrow \chi \chi)$ and the spin-independent $\chi$-proton elastic cross section $\sigma_{\chi p}^{\text {SI }}$ can be easily calculated in terms of the parameters of the Lagrangian, and 
we refer to Ref. [11] for complete expressions. For the present purpose, it is important that both $\Gamma(H \rightarrow \chi \chi)$ and $\sigma_{\chi p}^{\mathrm{SI}}$ are proportional to $\lambda_{H \chi \chi}^{2}$; therefore, the ratio $r_{\chi}=\Gamma(H \rightarrow \chi \chi) / \sigma_{\chi p}^{\mathrm{SI}}$ depends only on the dark matter mass $M_{\chi}$ and known masses and couplings. This allows us to relate the invisible Higgs branching fraction to the direct detection cross section:

$$
\mathrm{BR}_{\chi}^{\mathrm{inv}} \equiv \frac{\Gamma(H \rightarrow \chi \chi)}{\Gamma_{H}^{\mathrm{SM}}+\Gamma(H \rightarrow \chi \chi)}=\frac{\sigma_{\chi p}^{\mathrm{SI}}}{\Gamma_{H}^{\mathrm{SM}} / r_{\chi}+\sigma_{\chi p}^{\mathrm{SI}}}
$$

with $\Gamma_{H}^{\mathrm{SM}}$ the total Higgs decay width into all particles in the SM. For a given $M_{\chi}$, the above formula connects the invisible branching fraction probed at the LHC to the dark matter-nucleon scattering cross section probed by XENON100. For $m_{p} \ll M_{\chi} \ll \frac{1}{2} M_{H}$, and assuming the visible decay width equals to the SM total width $\Gamma_{H}^{\mathrm{SM}}=4.0 \mathrm{MeV}$, one can write down the approximate relations in the three cases that we are considering [12].

Thus, for a given mass of dark matter, an upper bound on the Higgs invisible branching fraction implies an upper bound on the dark matter scattering cross section on nucleons. In Fig. 3 we show the maximum allowed values of the scattering cross section, assuming the $20 \%$ bound on $\mathrm{BR}_{\chi}^{\mathrm{inv}}$. Clearly, the relation between the invisible branching fraction and the direct detection cross section strongly depends on the spinorial nature of the dark matter particle, in particular, the strongest (weakest) bound is derived in the vectorial (scalar) case.

In all cases, the derived bounds on $\sigma_{\chi p}^{\text {SI }}$ are stronger than the direct one from XENON100 in the entire range where $M_{\chi} \ll \frac{1}{2} M_{H}$. In other words, the LHC is currently the most sensitive dark matter detection apparatus, at least in the context of simple Higgs-portal models (even more so if $\chi$ is a pseudoscalar, as in [13]). This conclusion does not rely on the assumption that the present abundance of $\chi$ is a thermal relic fulfilling the WMAP constraint of $\Omega_{D M}=0.226$ [14], and would only be stronger if $\chi$ constitutes only a fraction of dark matter in the universe. We also compared the bounds to the projected future sensitivity of the XENON100 experiment (corresponding to $60,000 \mathrm{~kg}-\mathrm{d}, 5-30 \mathrm{keV}$ and $45 \%$ efficiency).

Of course, for $M_{\chi}>\frac{1}{2} M_{H}$, the Higgs boson cannot decay into dark matter ${ }^{1}$, in which case the LHC cannot compete with the XENON bounds.

On the other hand, heavier dark matter, particularly for $M_{\mathrm{DM}} \gtrsim 80 \mathrm{GeV}$, is allowed by both $\mathrm{BR}^{\mathrm{inv}}$ and XENON100. We note that almost the entire available parameter space will be probed by the XENON100 upgrade. The exception is a small resonant region around $62 \mathrm{GeV}$, where the Higgs-DM coupling is extremely small. This can also be seen from Fig. 3, which displays predictions for the spin-independent DM-nucleon cross section $\sigma_{\mathrm{SI}}$ subject to the WMAP and $\mathrm{BR}^{\text {inv }}<10 \%$ bounds. The upper band corresponds to the fermion Higgs-portal DM and is excluded by XENON100. On the other hand, scalar and vector DM are both allowed for a wide range of masses. Apart from a very small region around $\frac{1}{2} m_{h}$, this parameter space will be probed by XENON100-upgrade and XENON1T. The typical value for the scalar $\sigma_{\mathrm{SI}}$ is a few times $10^{-9} \mathrm{pb}$, whereas $\sigma_{\mathrm{SI}}$ for vectors is larger by a factor of 3 which accounts for the number of degrees of freedom.

\footnotetext{
${ }^{1}$ In this case, one should consider the pair production of dark matter particles through virtual Higgs boson exchange, $p p \rightarrow H^{*} X \rightarrow \chi \chi X$. The rates are expected to be rather small [11].
} 

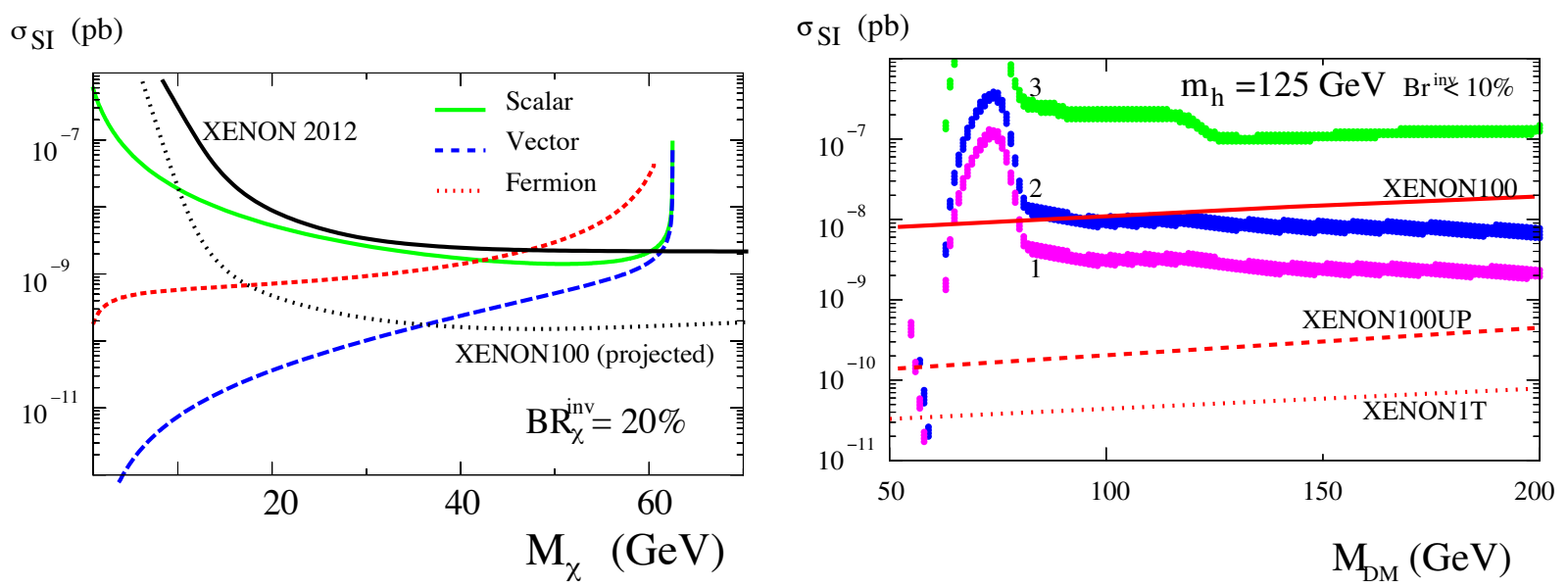

Figure 3: Left: Bounds on the spin-independent direct detection cross section $\sigma_{\chi p}^{\mathrm{SI}}$ in Higgs portal models derived for $M_{H}=125 \mathrm{GeV}$ and the invisible branching fraction of $20 \%$ (colored lines). For comparison, we plot the current and future direct bounds from the XENON experiment (black lines). Right: Spin independent DM-nucleon cross section versus DM mass. The upper band (3) corresponds to fermion DM, the middle one (2) to vector DM and the lower one (1) to scalar DM. The solid, dashed and dotted lines represent XENON100, XENON100 upgrade and XENON1T sensitivities, respectively.

\section{Conclusion}

The mass $M_{h} \approx 125 \mathrm{GeV}$ and the non-observation of SUSY particles, seems to indicate that the soft-SUSY breaking scale might be large, $M_{S} \gtrsim 1 \mathrm{TeV}$. We have discussed a simplified framework that describes the MSSM Higgs sector after the discovery of the lighter $h$ boson. Including the constraint $M_{h}=125 \mathrm{GeV}$, it can be again parameterized by only two inputs $\tan \beta$ and $M_{A}$. Allowing large $M_{S}$ values reopens the low $\tan \beta$ region which can be probed in many interesting processes at the LHC. In a second step, we have shown that to describe accurately the $h$ properties when the direct radiative corrections are also important, the three couplings $c_{t}, c_{b}$ and $c_{V}$ are needed besides the $h$ mass. We have performed a fit of these couplings using the latest LHC data. In the limit of heavy sparticles (i.e. with small direct corrections), the best fit point will be accessible in the next LHC run.

In a second part, we analyzed in a model-independent way the interplay between the invisible Higgs branching fraction and the dark matter scattering cross section on nucleons, in the context of effective Higgs portal models. The limit $\mathrm{BR}_{\mathrm{inv}}<0.2$, suggested by the combination of Higgs data in the visible channels, implies a limit on the direct detection cross section that is stronger than the current bounds from XENON100, for scalar, fermionic, and vectorial dark matter alike. Hence, in the context of Higgs-portal models, the LHC is currently the most sensitive dark matter detection apparatus and we find that light Higgs-portal DM $M_{\mathrm{DM}} \lesssim 60 \mathrm{GeV}$ is excluded independently of its nature. We also find that the entire class of Higgs-portal DM models will be probed by the XENON100-upgrade and XENON1T direct detection experiments, which will also be able to discriminate between the vector and scalar cases. The fermion DM is essentially ruled out by the current data, most notably by XENON100. 


\section{References}

[1] L. Maiani, A. D. Polosa and V. Riquer, New J. Phys. 14 (2012) 073029 [arXiv:1202.5998 [hep-ph]].

[2] A. Djouadi and J. Quevillon, JHEP 1310 (2013) 028 [arXiv:1304.1787 [hep-ph]].

[3] J. Quevillon, arXiv:1405.2241 [hep-ph].

[4] A. Djouadi, L. Maiani, A. Polosa, J. Quevillon and V. Riquer, arXiv:1502.05653 [hep-ph].

[5] A. Arbey, M. Battaglia, A. Djouadi, F. Mahmoudi and J. Quevillon, Phys. Lett. B 708 (2012) 162 [arXiv:1112.3028 [hep-ph]].

[6] A. Djouadi, L. Maiani, G. Moreau, A. Polosa, J. Quevillon and V. Riquer, Eur. Phys. J. C 73 (2013) 2650 [arXiv:1307.5205 [hep-ph]].

[7] A. Djouadi and G. Moreau, Eur. Phys. J. C 73 (2013) 9, 2512 [arXiv:1303.6591 [hep-ph]].

[8] G. Aad et al. [ATLAS Collaboration], JHEP 1302 (2013) 095 [arXiv:1211.6956 [hep-ex]].

[9] CMS Collaboration, CMS-PAS-HIG-13-021.

[10] T. Hambye and M. H. G. Tytgat, Phys. Lett. B 683, 39 (2010) [arXiv:0907.1007 [hep-ph]]; S. Kanemura, S. Matsumoto, T. Nabeshima and N. Okada, Phys. Rev. D 82 (2010) 055026 [arXiv:1005.5651]; O. Lebedev, H. M. Lee and Y. Mambrini, Phys. Lett. B 707 (2012) 570 [arXiv:1111.4482 [hep-ph]]; J. F. Kamenik and C. Smith, arXiv:1201.4814 [hep-ph]; T. Nabeshima, arXiv:1202.2673.

[11] A. Djouadi, O. Lebedev, Y. Mambrini and J. Quevillon, Phys. Lett. B 709 (2012) 65 [arXiv:1112.3299 [hep-ph]].

[12] A. Djouadi, A. Falkowski, Y. Mambrini and J. Quevillon, Eur. Phys. J. C 73 (2013) 6, 2455 [arXiv:1205.3169 [hep-ph]].

[13] L. Lopez-Honorez, T. Schwetz and J. Zupan, arXiv:1203.2064 [hep-ph].

[14] E. Komatsu et al. [WMAP Collaboration], Astrophys. J. Suppl. 192 (2011) 18 [arXiv:1001.4538]. 